\title{
Efficient electro encephelogram classification system using support vector machine classifier and adaptive learning technique
}

\author{
Virupaxi Balachandra Dalal ${ }^{1}$, Satish S. Bhairannawar ${ }^{2}$ \\ ${ }^{1}$ Department of Electronics and Communication Engineering, Jain College of Engineering and Research, Belagavi, India \\ ${ }^{2}$ Department of Electronics and Communication Engineering, SDM College of Engineering and Technology, Dharwad, India
}

\begin{tabular}{l} 
Article Info \\
\hline Article history: \\
Received Jul 13, 2021 \\
Revised Oct 21, 2021 \\
Accepted Nov 27, 2021 \\
\hline
\end{tabular}

Keywords:

Adaptive learning

Classifier

Electro encephelogram

Spatial filter

Support vector machine

\begin{abstract}
Complex modern signal processing is used to automate the analysis of electro encephelogram (EEG) signals. For the diagnosis of seizures, approaches that are simple and precise may be preferable rather than difficult and time-consuming. In this paper, efficient EEG classification system using support vector machine (SVM) and Adaptive learning technique is proposed. The database EEG signals are subjected to temporal and spatial filtering to remove unwanted noise and to increase the detection accuracy of the classifier by selecting the specific bands in which most of the EEG data are present. The neural network based SVM is used to classify the test EEG data with respect to training data. The cost-sensitive SVM with proposed Adaptive learning classifies the EEG signals where the adaptive learning with probability based function helps in prediction of the future samples and this leads in improving the accuracy with detection time. The detection accuracy of the proposed algorithm is compared with existing which shows that the proposed algorithm can classify the EEG signal more effectively.
\end{abstract}

This is an open access article under the CC BY-SA license.

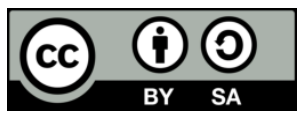

\section{Corresponding Author:}

Virupaxi Balachandra Dalal

Department of Electronics and Communication Engineering, Jain College of Engineering and Research

Belagavi, Karnataka, India

Email: virupaxi.dalal@gmail.com

\section{INTRODUCTION}

The electroencephalogram (EEG) is one of the most common and straightforward methods for detecting and diagnosing seizures. The ease with which EEG could be used by ordinary people on a budget made it a popular method. The EEG data acquired from the brain are also complex due to the sophisticated architecture of the brain system. The electrical signal generated by the human brain is captured through the electrode attached to a person's head. Due to the advancement of the computer based processing algorithm, now a days it is possible to develop automatic classification of EEG signals. In this paper, we developed efficient neural network based EEG classification system which is implemented and tested using standard EEG dataset on matrix laboratory (MATLAB) tool. Given recent improvements in mobile apps, EEG technologies signified the historical beginning of human neuroscience research to analyze brain activity [1], and they currently stand firmly as one of the most accessible and versatile instruments to study the brain in real-time. We highlighted important hurdles in completely integrating both relevant hardware and software improvements into neurodevelopmental research for mobile EEG devices. There are several obstacles to overcome if mobile EEG developments are to be fully utilized in neurodevelopmental research. Hardware and software advancements are certainly moving at a breakneck pace. It has been suggested that we can use EEG to construct our own data base for emotion analysis. In research on emotion estimate, 16-32 electrodes were employed during EEG recording [2], which is a time and processing speed constraint. During EEG 
recording, fewer electrodes can be used. Due to its impressive findings, the extreme learning machine (ELM) has risen to the forefront of classification. The most essential characteristic of this model is that it speeds up the learning process. ELM was not employed in any of the investigations that used EEG to classify emotion recognition. It is advised that for the classification of emotion recognition processes using EEG, only employ ELM or combine it with other approaches.

Craik et al. [3] performs a review on different EEG classification techniques. For this purpose, deep learning based techniques such as recurrent neural networks (RNN), sparse auto-encoders (SAE) neural networks, multi-layer perceptron (MLP), and hybrid techniques. The hybrid technique generates better result than standalone technique. Ilyas et al. [4] performs performance comparisons of different types of EEG signals. For this purpose, the authors considered support vector machine (SVM), k-nearest neighbour (k-NN), multilayer perceptron artificial neural network (MLP-ANN) and logistic regression (LR) techniques using brain-computer interface (BCI) competition IV-Dataset 1. Among those techniques, LR and SVM techniques shows better accuracy than other techniques. Nagabushanam et al. [5] proposed long-short term memory (LSTM) and improved neural network based EEG signal classification. The existing neural network algorithm is modified to get optimized accuracy. The gradient based function with radial basis operations are used for implementation. The performance is analyzing using Python in keras. Shi et al. [6] presented squirrel search algorithm with SVM for efficient EEG classification. To analyze the performance, the braincomputer interface (BCI) competition 2003 dataset III is used which proves that the proposed technique is better than existing. Dai et al. [7] presented EEG classification system based on deep learning techniques. In this paper, the variational auto encoder (VAE) technique is combined with the general convolutional neural network $(\mathrm{CNN})$ algorithm to get good classification. The performance of the system is evaluated using BCI competition IV dataset $2 \mathrm{~b}$ which shows the increment in detection accuracy than existing. Kumar et al. [8] presented EEG classification system based on optical predictor on different memory based network.

In this paper they presented a new classification method to predict the EEG signal which is the combination of common spatial pattern (CSP) and long short-term memory (LSTM) network. This technique is tested on different standard dataset. Tahir et al. [9] presented EEG classification using swarm optimization with neural network for epilepsy detection. The algorithm is implemented on MATLAB tool and simulated with standard EEG databases. The simulation result shows that testing accuracy is very high for the proposed technique. A clear understanding of various signal processing techniques is described in this paper [10]. This survey will give the researchers a way to select the appropriate signal processing method according to their need. EEG classification using deep learning approach is still in its primary stages. There is a need to find architecture and training paradigms for deep earning methods in order to improve classification. However, as research continues into the use of deep learning for classification of EEG signals, best practices will become well known.

In this work [11], a novel solution to the problem of EEG signal feature extraction and categorization is presented. The electrodes are first screened depending on their relevance, then the wavelet transform is performed to extract EEG features, and finally an AlexNet classifier is applied. EEG signals can be properly extracted and classified using the method described above. Furthermore, the results show that the suggested method uses little resources and has a high degree of accuracy. As a result, this strategy is extremely useful for resolving difficulties of this nature. The SNR has to be improved because artefacts distort the EEG signals. This aids in enhancing feature extraction and data categorization accuracy presented in paper [12]. As a result, recent strategies are examined, together with their benefits and drawbacks. It has been discovered that the procedures outlined in the review produce better results than traditional methods. The cerebral cortex generates EEG signals, which are always tainted by certain disturbances [13]. Despite the fact that a variety of strategies for removing unwanted artefacts have been created, an artefact removal method that combines high accuracy with algorithmic efficiency has yet to be established. Based on insights drawn from the literature, this report described the key strategies. Each method's pros and disadvantages are also discussed. There is no one-size-fits-all solution for removing all forms of artefacts. As a result, one of the long-term goals of effective artefact attenuation is to create an application-specific algorithm that is more efficient in terms of time and accuracy. Also, based on the current artefact removal trend, future directions will integrate machine learning and classical methodologies for successful automatic artefact removal. A process for exploiting feature extraction and classification techniques to EEG signals is presented in this paper [14], [15]. Specifically, a time-frequency analysis using both the fourier and wavelet transforms is done on 109 samples from the Alzheimer's disease (AD), mild cognitive impairment (MCI) and hierarchical classification (HC) classes. The following phases are included in the classification procedure: i) EEG signal preprocessing; (ii) feature extraction using the discrete fourier and wavelet transforms; and (iii) classification using tree-based supervised algorithms. We discover that Wavelets analysis outperforms fourier analysis when comparing classification performance with both feature extraction approaches. In this paper we proposed an efficient EEG classification system using SVM and adaptive learning technique combinedly. 


\section{PROPOSED ALGORITHM}

The proposed EEG classification system is shown in Figure 1 which consists of temporal filtering, spatial filtering, SVM classifier, cost-sensitive SVM (CSVM) validation and adaptive learning respectively. The training and test dataset are filtered using temporal filtering which are then enhanced using spatial filter and send to SVM Classifier. The classified signals are then validate using CSVM validation with the help of adaptive learning block.

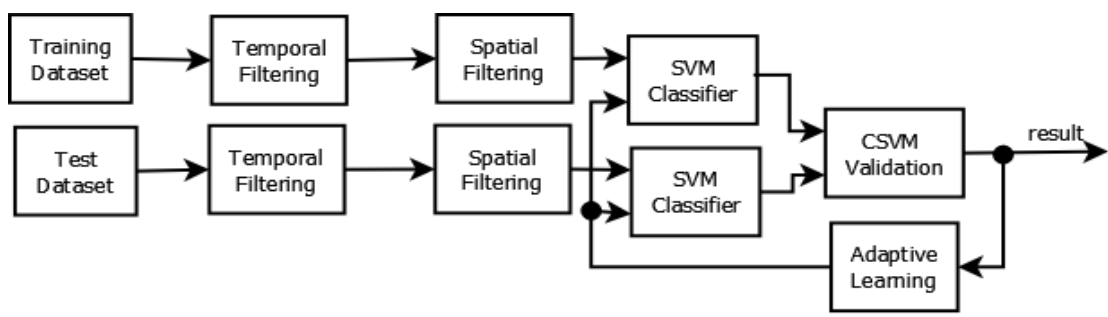

Figure 1. Proposed EEG system classification

\subsection{Dataset}

The BCI competition IV dataset [16] is used as standard database for training and test. For our implementation, dataset $2 \mathrm{~A}$ is considered which consists of 9 subjects captured using 22 EEG channels and 3 monopolar electrooculogram (EOG) channel as shown in Figure 2 [17]. The four motor imagery tasks in the cue-based BCI paradigm were imagination of movement of the left hand (class 1), right hand (class 2), both feet (class 3), and tongue (class 4). On two separate days, two sessions were held. Each subject was recorded. Each session consists of six runs separated by a minute. Breaks should be brief. One run consists of 48 trials (12 for each of the four possible classes), yielding a total of 288 trials per session.

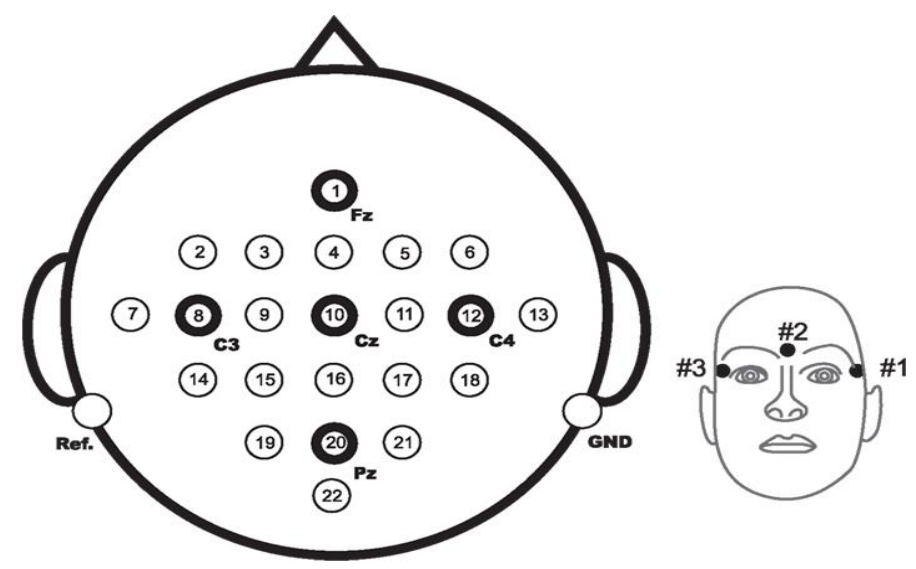

Figure 2. Classification electrode placing for EEG signal capture

\subsection{Temporal filter}

The captured electronic signals through electrode are analogues in nature and consist of different frequency bands. Among those frequency bands, only the $8-12 \mathrm{~Hz}$ and $14-30 \mathrm{~Hz}$ frequency bands data are mainly used for EEG processing purpose due to its stable response. Those bands are generally known as $\mu$ band and $\beta$-band respectively [18] which are separated using temporal filter from the standard EEG signal. The standard band-pass filter is used as temporal filter with suitable coefficients [19].

\subsection{Spatial filter}

Any raw EEG signal consists of very low spatial resolutions which make it difficult to classify EEG signal efficiently. In many cases, it creates difficulties to analyze the rhythm patterns generated by the brain signals which are generally known as event-related desynchronization (ERD) or event-related synchronization (ERS) [20]. The common spatial pattern (CSP) algorithm is normally used to detect such scenarios. To get optimized result, the existing algorithm is modified. As a result, the overall time domain signal is concatenated into smaller time based segments and decomposes the overall signal into a finite set represented as (1), 
$W \in R^{c(t) \times c(t)}$

where, $\mathrm{c}(\mathrm{t})$ is the signal from the respective channel at a finite time span.

For single trial EEG signal, this equation can be rewritten as (2),

$Z \in R^{c(t)}$

The relations between signal spaces in different trials are,

$$
Z=W \cdot E
$$

where, E is the EEG measurement data of a single trail.

To get effective measurement of different mental state features, it is essential to make the variance is very small. For such cases, the feature vectors can be written as (4),

$$
x_{t}=\log \left(\frac{\sigma_{z(t)}}{\sum_{i=1}^{2 n} \sigma_{z_{i}(t)}}\right)
$$

where, $\mathrm{n}$ is one row of the matrix signal.

\subsection{SVM classifier and validation}

The support vector machine (SVM) uses learning method based on statistical properties of the input signal. The separate hyper-planes are mathematically derives from those properties with maximum margin. The equations used to generate optimum hyper-plane are,

$$
\omega . x_{i}+\text { bias }=\left\{\begin{array}{l}
\geq+1 ; \text { if } y_{i}=+1 \\
<+1 ; \text { if } y_{i}=-1
\end{array}\right.
$$

where, $x i$ is the ith input vector.

yi is the assigned level of the ith input.

$\omega$ is the weight vector.

The optimal margin separating different hyper-planes can be written as [21].

$$
\omega x_{i}+\text { bias } \leq \pm 1
$$

The support vectors are then calculated through the transform space,

$$
k\left(x_{i}, x_{j}\right)=\varphi(x) \varphi\left(x_{j}\right)
$$

For the above equation, the feature class calculation equation can be written as (8),

$$
f(x)=\operatorname{sign}\left(\sum_{i} \alpha_{i} y_{j} \varphi(x) \varphi\left(x_{i}\right)+\text { bias }\right)
$$

where, $\alpha$ is dynamic weight factor.

\subsection{Adaptive learning}

The features generated by the classifier are then used to predict the classes of future samples depending upon the training dataset and test dataset. The efficient classification is generated by this method. In our case we consider the probability distribution function to predict the future samples. To make the classification effective the following equations are used.

The equation of training dataset in adaptive learning condition can be defined as (9),

$$
X_{\text {Train }}=\prod_{i=1}^{N}\left\{x_{y}, y_{i}\right\}
$$

where, $\mathrm{N}$ is the total observation number; $\mathrm{y}_{\mathrm{i}}$ is the level assigned for respective $\mathrm{xi}$ input.

Since for the classification only two classes are considered, so we can write,

$$
\mathrm{y} \in\left\{\begin{array}{l}
\mathrm{c}_{1} ; \text { if } \mathrm{x}_{\mathrm{i}} \text { belongs to class } \omega_{1} \\
\mathrm{c}_{2} ; \text { if } \mathrm{x}_{\mathrm{i}} \text { belongs to class } \omega_{2}
\end{array}\right.
$$

For SVM, we have considered the class value is \pm 1 in section 3 . So, the probability distribution function of the input signal can be written as (11), 


$$
\mathrm{p}\left(\mathrm{x}_{\mathrm{i}}\right)=\mathrm{p}\left(\omega_{1}\right) \cdot \mathrm{p}\left(\mathrm{x}_{\mathrm{i}} \mid \omega_{1}\right)+\mathrm{p}\left(\omega_{2}\right) \cdot \mathrm{p}\left(\mathrm{x}_{\mathrm{i}} \mid \omega_{2}\right)
$$

Where, $p\left(\oplus_{1}\right)$ and $p\left(\omega_{2}\right)$ are the probability of the respective class; $\mathrm{p}\left(\mathrm{x}_{\mathrm{i}} \mid \omega_{1}\right)$ and $\mathrm{p}\left(\mathrm{x}_{\mathrm{i}} \mid \omega_{2}\right)$ are the probability distribution function.

The predicted future sample is then (12),

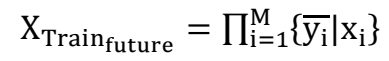

where, $\mathrm{M}$ is observation in testing; $\overline{\mathrm{y}_{\mathrm{i}}}$ is the predicted sample.

\section{SIMULATION RESULT}

The proposed algorithm is simulated on MATLAB 2017a software using standard programming method. For this simulation, standard BCI competition IV dataset are used. The features generated by the proposed algorithm is shown in the Figure 3 and Figure 4 for different frequency bands which is classified into two different data feature sets namely first and second best features. In this case the first best features are generated by considering the accurate feature sets of the corresponding frequencies and for the second best feature sets which are very nearer to the accurate features are considered. The intersections of the first and second features are mainly used for a classification which helps to increase the detection rate. For the standard dataset [16] we obtained $85.714 \%$ accuracy in our proposed method.

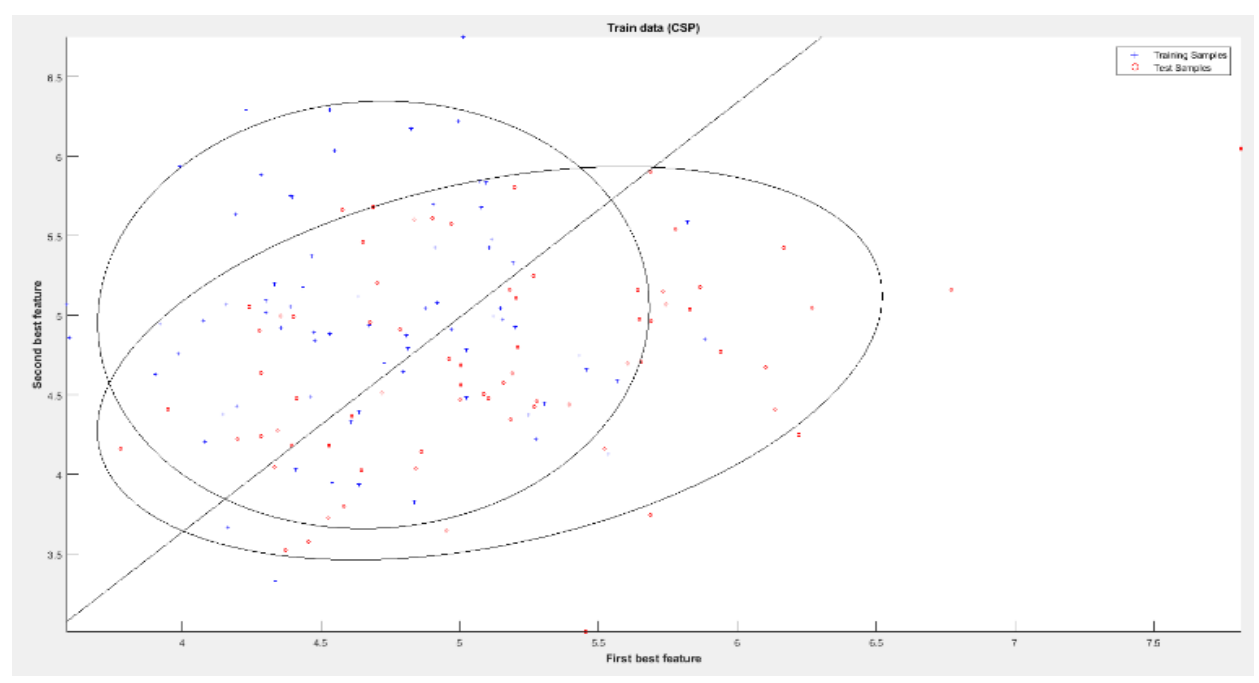

Figure 3. Feature set at $8-12 \mathrm{~Hz}$ band

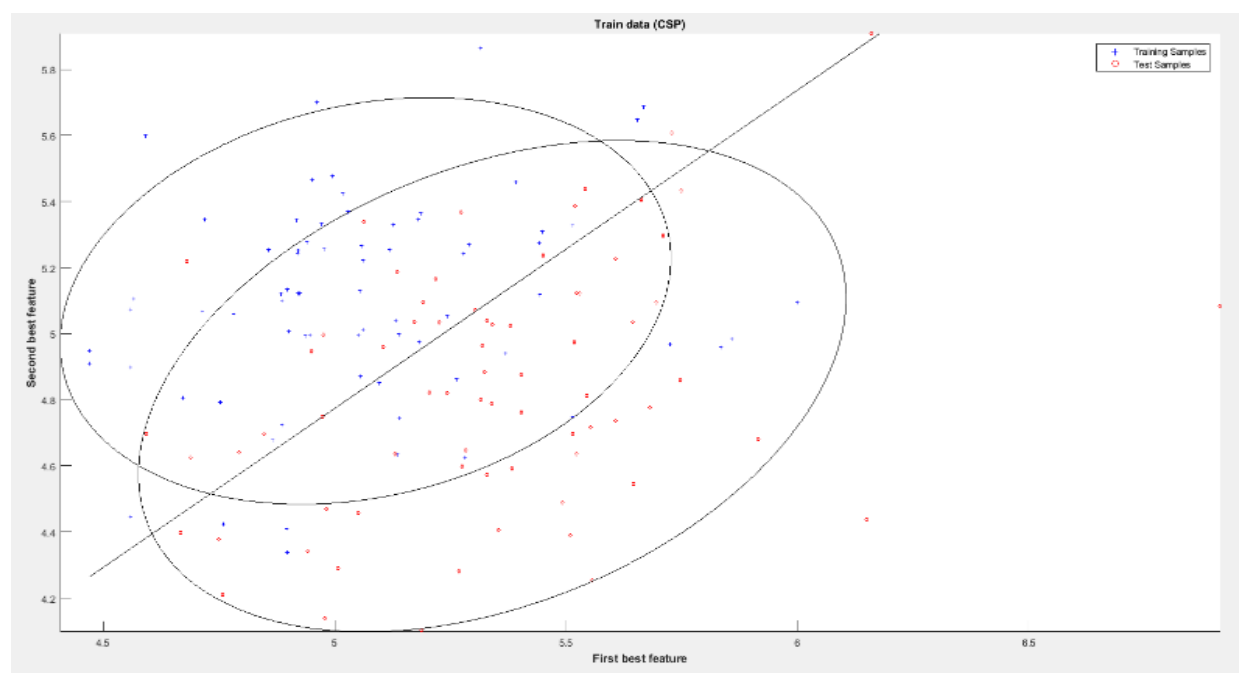

Figure 4. Feature set at $15-30 \mathrm{~Hz}$ band 


\section{RESULTS AND DISCUSSION}

The BCI competition IV dataset is used as standard database for training and test. For our implementation, dataset $2 \mathrm{~A}$ is considered which consists of 9 subjects captured using 22 EEG channels and 3 monopolar EOG channel. The true detection rate of the proposed algorithm is compared with the detection rate of various existing algorithms [22]-[26] in Table 1. It can be seen the existing refrences mentioned [22]-[26] uses simple SVM to multilayer perceptron and Fuzzy Logics without much focusing on Adaptive Learning. But, in our method that due to the use of SVM classification and Adaptive Learning techniques, the detection accuracy is higher in the case of proposed algorithm than existing techniques due to probability distribution based learning technique where it predicts future samples.

Table 1. True detection rate comparison

\begin{tabular}{llc}
\hline \multicolumn{1}{c}{ Authors } & \multicolumn{1}{c}{ Techniques } & Detection Rate \\
\hline Garrett et al. [22] & Basic SVM & $72 \%$ \\
Kerkeni et al. [23] & Multilayer Perceptron & $76 \%$ \\
Aljazaery et al. [24] & Quantum Neural Network & $81.33 \%$ \\
Nandish et al. [25] & Fuzzy Classifier & $80 \%$ \\
Bhatt and Gopal [26] & Fuzzy Classification & $76.2 \%$ \\
Proposed & SVM with Adaptive Learning & $85.714 \%$ \\
\hline
\end{tabular}

\section{CONCLUSION}

In this paper an efficient EEG classification algorithm is proposed which uses SVM classifier with adaptive learning techniques. The SVM technique is used to generate the feature vectors from the EEG data. The adaptive learning technique will optimize the features according to classes by using probability distribution function. The test and database data are then compared to get the detected vectors. To get accurate detections, the first best features and second best features are considered. To achieve more accuracy the adaptive learning techniques are used which changes the weights used in feature extractions. We obtained $85.714 \%$ accuracy with our proposed technique due to optimized classification of features into classes. In future the algorithm can be further improved interms of classification accuracy with time to classify all types of sub data sets available in the dataset.

\section{ACKNOWLEDGEMENTS}

Authors thanks to Department of Electronics and Communication Enggineering, SDMCET, Dharwad and Department of Electronics and Communication Engineering, Jain College of Engineering and Research, Belagavi, Karnataka, India, for providing an infrastructure to carry research on above mentioned topic.

\section{REFERENCES}

[1] A. Lau-Zhu, M. P. H. Lau, and G. McLoughlin, "Mobile EEG in research on neurodevelopmental disorders: Opportunities and challenges," Developmental Cognitive Neuroscience, vol. 36, pp. 1-14, Apr. 2019, doi: 10.1016/j.dcn.2019.100635

[2] N. Yoldorom and A. Varol, "A Research on Estimation of Emotion Using EEG Signals and Brain Computer Interfaces," in Proc. $2^{\text {nd }}$ International conference on computer science and engg IEEE, 2017, doi: 10.1109/UBMK.2017.8093523.

[3] A. Craik, Y. He, and J. L. Contreras-Vida, "Deep learning for electroencephalogram(EEG) classification tasks: a review," IOP Journal of Neural Engineering, vol. 16, no. 3, pp. 1-28, 2019, doi: 10.1088/1741-2552/ab0ab5.

[4] M. Z. Ilyas, P. Saad, M. I. Ahmad, and A. R. I. Ghani, "Classification of EEG Signals for Brain-Computer Interface Applications: Performance Comparison," IEEE International Conference on Robotics, Automation and Sciences (ICORAS), 2016, pp. 1-4, doi: 10.1109/ICORAS.2016.7872610.

[5] P. Nagabushanam, S. T. George, and S. Radha, "EEG signal classification using LSTM and improved neural network algorithms," Methodologies anf Application, vol. 24, pp. 9981-10003, 2020, doi: 10.1007/s00500-019-04515-0.

[6] M. Shi, C. Wang, X.-Z. Li, M.-Q. Li, L. Wang, and N.-G. Xie, "EEG signal classification based on SVM with improved squirrel search algorithm," International Journal of Biomedical Engineering / Biomedizinische Technik, vol. 66, no. 2, pp. 137-152, Sept. 2020, doi: 10.1515/bmt-2020-0038.

[7] M. Dai, D. Zheng, R. Na, S. Wang, and S. Zhang, "EEG Classification of Motor Imagery Using a Novel Deep Learning Framework," Sensors, vol. 19, pp. 1-16, Jan. 2019, doi: 10.3390/s19030551.

[8] S. Kumar, A. Sharma, and T. Tsunoda, "Brain wave classifcation using longshort-term memory network based Optical predictor," International Journal of Nature, vol. 9, pp. 1-13, June 2019, doi: 10.1038/s41598-019-45605-1.

[9] I. Tahir, U. Qamar, H. Abbas, B. Zeb, and S. Abid, "Classification of EEG Signal by Training Neural Network with Swarm Optimization for Identification of Epilepsy," in Proc. $11^{\text {th }}$ IEEE International Conference on Machine Learning and Computing, Feb. 2019, pp. 197-203, doi: 10.1145/3318299.3318374.

[10] M. Iftikhar, S. A. Khan, and A. Hassan, "A Survey of Deep Learning and Traditional Approaches for EEG Signal Processing and Classification," in Proc. IEEE $9^{\text {th }}$ Annual Information Technology, Electronics and Mobile Communication Conference (IEMCON), 2018, pp. 395-400, doi: 10.1109/IEMCON.2018.8614893. 
[11] S. Li and H. Feng, "EEG Signal Classification Method Based on Feature Priority Analysis and CNN," in Proc. International Conference on Communications, Information System and Computer Engineering (CISCE), 2019, pp. 403-406, doi: 10.1109/CISCE.2019.00095.

[12] R. S. Mahamune and S. H. Laskar, "A review on artifacts removal techniques for Electroencephalogram signals," in Proc. $2^{\text {nd }}$ International Conference on Innovations in Electronics, Signal Processing and Communication (IESC), 2019, doi: 10.1109/IESPC.2019.8902363.

[13] X. Jiang, G.-B. Bian, and Z. Tian, "Removal of Artifacts from EEG Signals: A Review," Sensors, vol. 19, no. 5, p. 987, 2019, doi: $10.3390 / \mathrm{s} 19050987$

[14] G. Fiscon et al., "Combining EEG signal processing with supervised methods for Alzheimer's patients classification," BMC Med Inform Decis Mak, vol. 18, no. 1, p. 35, May 2018, doi:10.1186/s12911-018-0613-y.

[15] S. Li and H. Feng, "EEG Signal Classification Method Based on Feature Priority Analysis and CNN," in Proc. International Conference on Communications, Information System and Computer Engineering (CISCE), 2019, pp. 403-406, doi: 10.1109/CISCE.2019.00095.

[16] BCI Competition IV, http://www.bbci.de/competition/iv/

[17] R. Leeb, F. Lee, C. Keinrath, and R. Scherer, "Brain-Computer Communication: Motivation, Aim, and Impact of Exploring a Virtual Apartment," IEEE transaction on neural systems and rehabilitation engineering: a publication of the IEEE engineering in Medicine and Biology Society, vol. 15, no. 4, 2008, doi: 10.1109/TNSRE.2007.906956.

[18] S. Siuly, Y. Li, and Y. Zhang, "EEG Signal Analysis and Classification," Springer, 2020, doi: 10.1007/978-3-319-47653-7.

[19] J. Baranowsk and P. Piątek, "Fractional Band-Pass Filters: Design, Implementation and Application to EEG Signal Processing," Journal of Circuits, Systems and Computers, vol. 26, no. 11, pp. 1-21, 2017, doi: 10.1142/S0218126617501705.

[20] K. K. Ang, Z. Y. Chin, C. Wang, C. Guan, and H. Zhang, "Filter bank common spatial pattern algorithm on BCI competition IV Datasets 2a and 2b," Journal of frontier in neuroscience, vol. 6, no. 39, pp. 1-9, 2012, doi: 10.3389/fnins.2012.00039.

[21] C. M. Bishop, Neural Networks for Pattern Recognition, Oxford University Press, Cambridge, 1995.

[22] D. Garrett, D. A. Peterson, C. W. Anderson, and M. H. Thaut, "Comparisonof linear and nonlinear methods for EEG signal classification," IEEE Transactions on Neural Systems and Rehabilitation Engineering, vol. 11, pp. 141-144, 2003, doi: 10.1109/TNSRE.2003.814441.

[23] N. Kerkeni, F. Alexandre, H. M. Bedoui, L. Bougrain, and M. Dogui, “Automatic classification of sleep stages on a EEG signal by artificialneural networks," in Proc. $5^{\text {th }}$ WSEAS Int. Conf. on Sig Speech And Image Processing, 2005, pp. 128-131.

[24] I. A. Aljazaery, A. A. Ali, and H. M. Abdulridha, "Classification of electroencephalograph (EEG) signals using quantum neural network," Signal Processing: An International Journal (SPIJ), vol. 4, no. 6, 2011.

[25] M. Nandish, M. Stafford, P. H. Kumar, and F. Ahmed, "Feature Extractionand Classification of EEG Signal Using Neural Network BasedTechniques," International Journal of Engineering and Innovative Technology (IJEIT), vol. 2, no. 4, Oct. 2012.

[26] R. B. Bhatt and M. Ghopal, "FRID: fuzzy-rough interactive dichotomizers," in Proc. IEEE International Conference on Fuzzy Systems, 2004, pp. 1337-1342, doi: 10.1109/FUZZY.2004.1375363.

\section{BIOGRAPHIES OF AUTHORS}

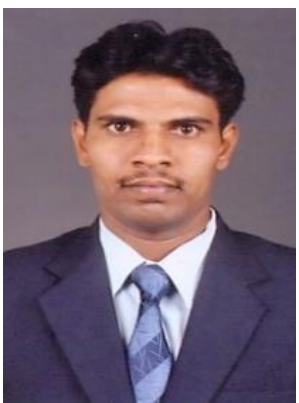

Virupaxi Balachandra Dalal (D) $8 \mathrm{SC}$ S is a research scholar at Department of Electronics and Communication Enggineering, SDMCET, Dharwad, Karnataka, India. Author is also working as Assistant Professor in Department of Electronics and Communication at Jain College of Engineering and Research, Belagavi, Karnataka, India. Author's area of intrest is Signals Processing and Image Processing. He can be contacted at email: virupaxi.dalal@gmail.com.

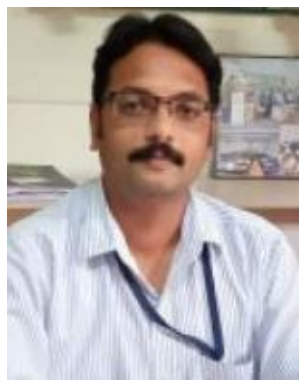

Dr. Satish S. Bhairannawar (iD) SC $\mathrm{P}$ holds the position of Dean of Industry Institute Interface (III), Chairperson, CEIR Startup incubation and Professor of Electronics \& Communication Engineering, SDMCET, Dharwad. He has been involved in research activities funded by reputed state and central govt. organizations and has published over 30 research publications in SCI, SCIE and Scopus Indexed Journal. He can be contacted at email: satishbhairannawar@gmail.com. 